\title{
Phase Modeling of Indoor Radio Propagation Channels
}

\author{
Homayoun Nikookar, Member, IEEE, and Homayoun Hashemi, Senior Member, IEEE
}

\begin{abstract}
Two models for generating phase of individual multipath components in an indoor environment, partly developed in [1], have been studied in detail. In the deterministic phase increment model (model I), phase of each multipath component is updated deterministically using several independent random scatterers. In the random phase increment model (model II), phase of each multipath component is updated by adding independent random phase increments. Performance of these models has been evaluated by means of extensive computer simulations. Statistical properties of narrow-band CW fading signals obtained using each phase model have been compared with the corresponding results for a large empirical wide-band database of 12000 impulse response estimates of indoor radio propagation channels. A major conclusion is that model I (with five scatterers for each multipath component) and model II (with proper choice for phase increments) provide fading results consistent with those obtained from measurements.

In this paper, properties of each phase model are described, and an algorithm for generating each is presented. First- and second-order statistics [amplitude distributions, level crossing rates (LCR's), and average duration of fades (ADF's)] and Doppler spectra of narrow-band $\mathrm{CW}$ fading waveforms obtained using simulated phases are reported, and detailed comparison between the simulated and empirical results is carried out. Furthermore, the two models are also compared with each other, and advantages and disadvantages of each are explored. The effect of increasing the number of scatterers and statistical properties of phase increments in model I are studied, simulated, and compared with model II. A major conclusion is that for an appropriate choice of parameters, both models provide satisfactory performance. Computation time of model I is, however, on the average 1.7 times of model II, making it less efficient for generating a large number of impulse response profiles.

The results reported in this paper can be used in performance evaluation of wireless indoor communication systems, either directly or by developing a comprehensive channel simulator.
\end{abstract}

Index Terms-Indoor propagation channel, multipath fading, phase modeling.

\section{INTRODUCTION}

I NDOOR radio communication systems provide wireless transmission of voice and data to people on the move inside

Manuscript received May 11, 1995; revised November 18, 1998. This work was initiated and supported by the Electrical Engineering Department, Sharif University of Technology, Tehran, Iran, and completed at the Telecommunications and Traffic Control Systems Group, Delft University of Technology, The Netherlands. This work was presented in part at the 44th annual IEEE Vehicular Technology Conference, Stockholm, Sweden, June 7-10, 1994.

H. Nikookar is with the International Research Center for Telecommunications, Transmission and Radar (IRCTR), Faculty of Information Technology and Systems, Department of Electrical Engineering, Delft University of Technology, 2600 GA Delft, The Netherlands.

H. Hashemi is with the Department of Electrical Engineering, Sharif University of Technology, Tehran, Iran.

Publisher Item Identifier S 0018-9545(00)02561-5. buildings. The associated services cover a wide variety of situations ranging from communication with individual walking in residential or office buildings, supermarkets, or shopping malls, etc., to fixed stations sending messages to robots in motion in assembly lines and factory environments of future.

Multipath fading seriously degrades performance of indoor radio communication systems. If the channel is well characterized, however, the effect of these disturbances can be reduced by proper design of transmitter and receiver. Therefore, detailed characterization of radio propagation is a major requirement for successful design of indoor wireless communication systems.

Measurements and modeling of indoor radio propagation channels have been reported in literature [1]-[16]. Reference [1] is a comprehensive tutorial-survey coverage of the topic. In [2], impulse response modeling of the indoor radio propagation channel has been investigated based upon a large database of 12000 impulse response estimates of the channel. Distribution of multipath components in each impulse response, distribution of arrival time of multipath components, correlation between path variables, and statistical properties of the rms delay spread are reported in [2] and [3]. Distribution of multipath components' amplitudes over both local and global areas is addressed in [2], and details are presented in [4].

Performance of digital communication systems operating in multipath environments is very sensitive to statistical properties of received signal's phase. In [17], empirical probability density function (pdf) of signal phase for a land mobile satellite channel is reported to be approximately Gaussian. References [6], [7], [18], and [19] have explored some aspects of phase modeling for the indoor (mobile) radio channels. Some simplifying assumptions have been made in these investigations. For example, in [6] and [7] it was assumed that over a length of $1 \mathrm{~m}$ in space, all multipath components with the same delay are caused by reflection from the same fixed scatterer. In [18] and [19], it was assumed that for small spatial separations the angle of arrival of each multipath component remains the same with respect to the direction of motion of vehicle (portable). To date, however, no empirically driven model for signal phase has been reported in the literature. This is probably due to difficulties associated with measuring the phase of individual multipath components. Although the above-mentioned references have addressed the issue of signal phase, they fall short of producing an elaborate phase model.

In this work, results of a comprehensive modeling effort to describe variations of received signal phase for digital transmission within buildings are reported. The results are based on mathematical analysis, simulation, and measurement. The large empirical database used in this project has been previously set 
up by the H. Hashemi. It consists of 12000 impulse response profiles of the channel collected at two office environments [2].

Although the techniques described in this report have been developed specifically for the indoor radio propagation channel, with proper modifications, the same ideas are applicable to other multipath environments, such as the outdoor vehicular mobile channel.

This paper is organized as follows. Section II provides background information, including a review of the mathematical modeling of the channel, and a brief description of the large empirical database used in subsequent analysis. Phase modeling of wireless indoor channel is carried out in Section III, and results of modeling are described in Section IV. In Section V, these phase models are compared with empirical data and with each other. Algorithms for generating these phase values are explained in Section VI, followed by concluding remarks of Section VII.

\section{BACKGROUND}

\section{A. Mathematical Model}

The random and complicated radio propagation channel can be characterized using the impulse response approach: at each point in a three-dimensional (3-D) environment, the channel is modeled as a time-varying linear filter with the impulse given by

$$
h(t, \tau)=\sum_{k=1}^{N(\tau)} a_{k}(t) \delta\left[t-\tau_{k}(t)\right] e^{j \theta_{k}(t)}
$$

where

$t \quad$ observation time of the impulse;

$\tau \quad$ application time of the impulse;

$N(\tau)$ number of multipath components;

$a_{k}(t)$ random time-varying amplitude;

$\tau_{k}(t)$ arrival time;

$\theta_{k}(t) \quad$ phase sequences;

$\delta \quad$ Dirac delta function.

The channel is completely characterized by these path variables. This mathematical model is a wide-band model, which has the advantage that because of its generality, it can be used to obtain the response of the channel to any transmitted signal $s(t)$ by convolving $s(t)$ with $h(t)$ and adding noise. It should he noted that, due to motion of people and equipment, the indoor radio propagation channel is, in general, time varying. However, during the "quiet hours," the time-invariant version of this model can be used as

$$
h(t)=\sum_{k=1}^{N} a_{k} \delta\left(t-\tau_{k}\right) e^{j \theta_{k}} .
$$

This model was first suggested by Turin [20] to describe multipath fading channels. It provided a consistent model for characterizing [21], [22] and simulating [23] the outdoor mobile radio channel. A convenient model for characterization of multipath propagation channels is the discrete-time impulse response model [12], [22], [23]. In this model, the time axis is divided into small time intervals called "bins." Each bin is assumed to contain either one multipath component or no multipath component. The possibility of more than one path in a bin is excluded. A reasonable bin size is the resolution of the specific measurement, since two paths arriving within a bin cannot be resolved as distinct paths. Using this model, each impulse response can be described by a sequence of "zeros" and "ones" (the path indicator sequence), where a "one" indicates the presence of a path in a given bin and a "zero" represents the absence of a path in that bin. To each "one," an amplitude and a phase value are associated.

\section{B. The Empirical Database}

A large empirical database of 12000 impulse response estimates of the channel previously collected at two dissimilar office environments was used to evaluate performance and range of applicability of each phase model. Details of the measurements and subsequent analysis of the data have been reported in [2] and [3]. A brief description of the database, which includes only relevant issues, is provided in this section.

In a typical wireless indoor communication system two-way transmission takes place between a fixed station and moving unit. Correspondingly, the following measurement plan was devised: four transmitter-receiver antenna separations of 5, 10, 20, and $30 \mathrm{~m}$ were considered. For each antenna separation several places for the base antenna were selected. The selection was made on the basis of what was considered to be typical positions for base antenna in future indoor systems. For each antenna separation, a total of 20 small $1.5-\mathrm{m}$ areas ("locations") was selected for the moving portable antenna position, The selections were made on the basis of good variation of typical conditions within the buildings. Each location was carefully chosen, with both line-of-sight (LOS) and nonline-of-sight (obstructed) topographies included. The number of locations with obstructed paths between the transmitter and receiver was higher for larger antenna separations, consistent with conditions encountered in real-life wireless indoor communication systems. For each location 75 response estimates of the channel recorded by displacing the portable antenna in steps of $2 \mathrm{~cm}$. It is important to emphasize that the measurement plan was entirely based on mathematical modeling of the channel described in the previous section.

The above measurement plan was executed at two dissimilar office buildings. Since the geometry of the buildings made it impossible to pick 20 portable locations per fixed site (base), the base sites were also varied for each antenna separation. This procedure brings the added advantage of making the results more general, as compared to scenario in which a single base station is selected for all measurements.

The first building (Bldg. A) was a T-shaped modern three-story facility containing hard partition offices, hallways, and laboratory space in addition to a number of soft-partitioned cubicles. Measurements were performed on the first and third floors, with both transmitter and receiver antennas located on the same floor. The second building (Bldg. B) was an octagon-shaped building with 27 floors. Measurements were carried out with both transmitter and receiver antennas on 19th floor only. The floor plan consists mainly of hard-partitioned hallways and soft-partitioned cubicles with standard office furniture [2]. 
The measurements were performed using a network analyzer to measure the frequency response of the indoor propagation channel between two discone antennas. In this method, the channel is excited with tones over a wide range of frequencies. The attenuation and phase shift of each frequency component (caused by propagation medium) are measured. The network analyzer swept frequency band for the measurements was $900-1300 \mathrm{MHz}$ in $500-\mathrm{KHz}$ steps (801 points). Base antenna height was $2.5 \mathrm{~m}$ for Bldg. A and $1.7 \mathrm{~m}$ for Bldg. B. The portable antenna height was $1.2 \mathrm{~m}$ for both buildings.

Due to motion of people and equipment indoor propagation channels are in general time varying. Since the purpose of these measurements was to investigate channel's variations in space (not in time), it was essential to keep the channel stationary during the measurements. All measurements were therefore performed at night or on weekends when there were few, if any, other personnel in the vicinity of measurement setup.

The 12000 measured frequency response data were later converted to time domain by classical Fourier analysis. Correspondingly, a large database of 12000 impulse response profiles was obtained. Resolution of time domain data was $5 \mathrm{~ns}$. The data were later reduced according to discrete-time impulse response model described in the previous section, with a bin size of $5 \mathrm{~ns}$. Details are reported in [2].

An examination of impulse responses for both buildings revealed that in most cases, multipath components were detectable only for excess delays (i.e., delays beyond the LOS delay) of less than $500 \mathrm{~ns}$.

\section{MODELING OF PHASE}

\section{A. General Comments}

Performance of digital indoor communication systems is very sensitive to statistical properties of the phase sequence $\left\{\theta_{k}\right\}$. Although the importance of this issue has been recognized by most investigators, a comprehensive search of the literature shows that, to date, no empirically driven model for the phase sequence has been reported [1].

The signal phase is critically sensitive to path length and changes by a factor of $2 \pi$ as the path length changes by a wavelength $(30 \mathrm{~cm}$ at $1 \mathrm{GHz})$. Considering the geometry of the paths, moderate changes (in order of meters) in the position of portable results in a great change in phase. When one considers an ensemble of points, therefore, it is reasonable to expect uniform distribution; i.e., on a global basis, $\theta_{k}$ has a $U[0,2 \pi)$ distribution. This phenomenologically reasonable assumption can be taken as a fact with no need for empirical verification. For small sampling distances, however, great deviations from uniformity may occur. Furthermore, phase values are strongly correlated if the channel's impulse response is sampled at the sampling rate (tens to hundreds of kilohertz). Phase values at a fixed delay for a given site are therefore correlated. Adjacent detectable multipath components of the same profile, on the other hand, have independent phases since their excess range (excess delay multiplied by speed of light) is longer than a wavelength, even for very high resolution (a few nanoseconds) measurements [1].

Taking the above into consideration it is reasonable to assume that the absolute phase value of a multipath component at a fixed point in space is not important; emphasis of the modeling should be placed on changes in phase as the portable moves through the channel. Let $\theta_{k}$ denote the phase of a multipath component at a fixed delay for profile number $k$, where $k=1,2, \cdots$, numbers adjacent points in space at a given site. Equivalently, $\theta_{k}$ may denote the phase of multipath component occupying a given bin (the discrete-time impulse response model) at spatial point $k$. For the first profile in a sequence $(k=1), \theta_{k}$ is assumed to have a $U[0,2 \pi)$ distribution. Subsequent phase values are assumed to follow the following relation [1]:

$$
\theta_{k}=\theta_{k-1}+\varphi\left(s_{k} / \lambda\right)
$$

where

$s_{k} \quad$ spatial separation between $(k-1)$ st and $k$ th profiles;

$\lambda \quad$ wavelength;

$\varphi\left(s_{k} / \lambda\right) \quad$ phase increment.

On a sequence of spatially separated profiles, the chain of values defined by (3) is interrupted when a path in a given excess delay time (or at a given bin) ceases to exist. A new chain of values (with uniformly distributed first component) starts if a path with the same excess delay appears at a later profile.

Appropriate choices for $\varphi\left(s_{k} / \lambda\right)$ will impose the necessary spatial correlation on phase values. Using this approach two models for this phase increment are considered [1].

\section{B. The Deterministic Phase Increment Model}

In the deterministic phase model (model I), changes in the phase value of a multipath component at a fixed delay when portable moves through space is not random; i.e., knowing $\theta_{1}$ and $\varphi\left(s_{k} / \lambda\right), \theta_{k}(k=2,3, \cdots)$ can be calculated deterministically.

A simplified version of this model has been used by some investigators to model spatial variations in the phase of a multipath component. In one such application, it was assumed that in a length of $1 \mathrm{~m}$ in space, all multipath components with the same delay are caused by reflection from the same fixed (but randomly located) scatterer [6], [7]. In this simulation application, the initial phase was generated according to a $U[0,2 \pi)$ distribution. Other spatially separated phases (with the same excess delay) were obtained by adding $\varphi\left(s_{k} / \lambda\right)$ to the previous phase value. The phase increment was calculated using the single scatterer and local geometry [6], [7]. This is a one-hop model which excludes multiple reflections. This phase model was used in a simulation package for predicting the impulse response of open plant and factory environments.

In two other simulation applications, the deterministic phase model has been used for the mobile and indoor channels [18], [19]. In both applications, it was assumed that the angle of arrival of the $n$th multipath component with respect to the direction of motion of vehicle (portable) $\psi_{n}$ remains the same for small spatial separations. Therefore

$$
\varphi\left(s_{k} / \lambda\right)=\frac{2 \pi s_{k}}{\lambda} \cos \psi_{n}
$$


For mobile channel $\psi_{n}(n=1,2,3, \cdots)$ were generated according to a uniform distribution. The power spectra of simulated $\mathrm{CW}$ data generated using a wide-band channel simulator (reported in [23]) showed better agreement with theory [18], when compared with the spectra obtained using the random phase increment model (which is described in Section III-C). For the indoor channel, $\psi_{n}(n=1,2,3, \cdots)$ were estimated with a $5^{\circ}$ resolution based on measurements and by using Fourier transform method (details are reported in [19]).

In the previous application of the deterministic phase increment model [6], [7], [18], [19], it was assumed that each multipath component results by reflection from a single reflector. This seems to be an oversimplification of the principal reality of the channel, since in practice there may be many indirect paths between transmitter and receiver for each multipath component. The resultant "subcomponents" will arrive with the same excess delay and add vectorially to produce one "distinct" resolvable multipath component. The deterministic phase increment model described in this work is, therefore, a multiple reflector model. The phase value of a multipath component of a given profile at a given excess delay is the phase of the phasor sum of individual contributing subcomponents. This phase value is updated deterministically as the portable (receiving) antenna moves through the channel.

The locus of all scatterers contributing to a single multipath component with a fix excess delay is an ellipse with the transmitter and receiver positioned at its focal points. Different confocal ellipses correspond to different excess delays. Scatterers are distributed uniformly on the perimeter of each ellipse.

The resolution of measured data is $\tau_{0}=5 \mathrm{~ns}$. In the discrete-time impulse response model each multipath component is assumed to arrive at the center of each bin. Adjacent (in time) components are, therefore, $\tau_{0}=5 \mathrm{~ns}$ apart. The corresponding adjacent ellipses have a "resolution" of $c \tau_{0} / 2=75 \mathrm{~cm}$, where $c$ is the speed of light and separation between two ellipses is defined as the difference between excess range (range beyond the LOS) of two rays reflected from two scatterers, located on two consecutive ellipses.

Referring to Fig. 1, portable moves from right focus of ellipses in the direction of positive main axis. The channel is sampled at the sampling distance $D$, which is $2 \mathrm{~cm}$ for the measured data. Considering the maximum excess delay of $N \tau_{0}$ and bin resolution of $5 \mathrm{~ns}$, the maximum number of confocal ellipses is $N=100$. Let $M$ denote the number of scatterers distributed on each ellipse. The $m$ th scatterer on the $n$th ellipse, $1 \leq m \leq M$, and $1 \leq n \leq N$ is labeled $S_{n, m}$. For a given position of portable and a fixed excess delay $M$ rays are received through reflection from the $M$ scatterers. As mentioned earlier, some ellipses do not exist because there is no multipath component at the corresponding-bin in the discrete-time impulse response. Therefore, $N$ is a random variable and, consequently, impulse response at the $k$ th spatial position is given by (2) with $h_{k}(t), a_{k, n}, \tau_{k, n}$, $\theta_{k, n}$, and $N_{k}$ replacing $h(t), a_{k}, \tau_{k}, \theta_{k}$, and $N$, respectively. $N_{k}$ is the number of multipath components in the $k$ th profile and $a_{k, n}, \tau_{k, n}, \theta_{k, n}$ are the random amplitude, time of arrival, and phase of the $n$th multipath component at the $k$ th profile, respectively. At the $k$ th position of portable, the amplitude and phase of $n$th multipath signal resulting from scatterers on $n$th

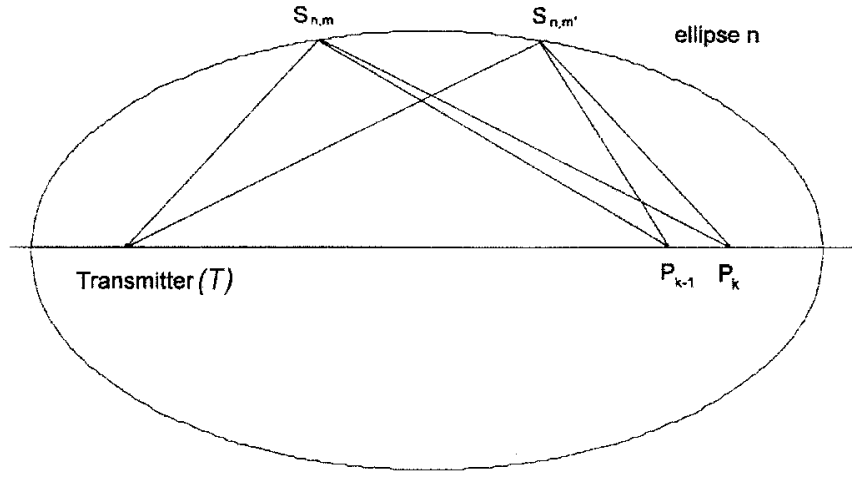

Fig. 1. Phase updating at a fixed excess delay in model I.

ellipse is

$$
R_{k, n} e^{j \phi_{k, n}}=\sum_{m=1}^{M} \alpha_{k, n, m} e^{j \theta_{k, n, m}}, \quad n=1,2, \cdots, N_{k}
$$

where $\alpha_{k, n, m}$ and $\theta_{k, n, m}$ are the real attenuation coefficient and phase shift, respectively (relative to the LOS path), of paths reflected from the $m$ th scatterer located on the $n$th ellipse, when portable is in its $k$ th position. $M$ is the number of scatterers on each ellipse and $R_{k, n}$ and $\phi_{k, n}$ are the amplitude and phase of the multipath component with excess delay $n \tau_{0}$. Using the geometry of Fig. $1, \theta_{k, n, m}$, the phase contribution of the $m$ th scatterer on the $n$th ellipse for the portable position $k$, is calculated from

$$
\theta_{k, n, m}=\theta_{k-1, n, m}+\frac{2 \pi}{\lambda}\left(\left|\overline{T S_{n, m} P_{k}}\right|-\left|\overline{T S_{n, m} P_{k-1}}\right|\right) .
$$

The second term of (6) can be represented as

$$
\Delta \beta_{k, n, m}=\frac{2 \pi}{\lambda}\left(\left|\overline{T S_{n, m} P_{k}}\right|-\left|\overline{T S_{n, m} P_{k-1}}\right|\right) .
$$

Therefore, (6) is rewritten as

$$
\theta_{k, n, m}=\theta_{k-1, n, m}+\Delta \beta_{k, n, m} .
$$

In Fig. 1, $T$ is the left focal point of the confocal ellipses, $P_{k}$ and $P_{k-1}$ are positions of the portable at $k$ th and $(k-1)$ st sampling distances, respectively, $S_{n, m}$ is the $m$ th scatterer on the $n$th ellipse, and $\lambda$ is the wavelength. The equation of the $n$th ellipse (i.e., the ellipse corresponding to the $n$th bin) is

$$
\frac{x^{2}}{p_{n}^{2}}+\frac{y^{2}}{q_{n}^{2}}=1
$$

Denoting the distance between the base and portable antennas by $2 r$ and the abscissa of the $m$ th scatterer by $x_{m}$, the value of $\Delta \beta_{k, n, m}$ in (7) is calculated as follows:

$$
\begin{aligned}
\Delta \beta_{k, n, m}=\frac{2 \pi}{\lambda}( & \sqrt{\left(r+K D-x_{m}\right)^{2}+q_{n}^{2}-c_{n}^{2} x_{m}^{2}} \\
& \left.-\sqrt{\left(r+K D-D-x_{m}\right)^{2}+q_{n}^{2}-c_{n}^{2} x_{m}^{2}}\right)
\end{aligned}
$$


where $c_{n}=q_{n} / p_{n}$ and $D$ is the sampling distance. Using (5), phase of the $n$th multipath component of the $k$ th profile is

$$
\phi_{k, n}=\tan ^{-1}\left(\frac{\sum_{m=1}^{M} \alpha_{k, n, m} \sin \theta_{k, n, m}}{\sum_{m=1}^{M} \alpha_{k, n, m} \cos \theta_{k, n, m}}\right) .
$$

The difference in phase of two multipath components occupying bin $n$ of two spatially adjacent impulse response profiles, $\varphi_{k, n}^{(I)}$ defined as $\phi_{k, n}-\phi_{k-1, n}$, is given by (12) at the bottom of the page, where superscript $(I)$ is used to indicate the phase change (i.e., the phase increment) of model I. Now the resultant CW envelope fading waveform at the $k$ th point in space is

$$
R_{k} e^{j \phi_{k}}=\sum_{n=1}^{N_{k}} R_{k, n} e^{j \phi_{k}, n} .
$$

The corresponding amplitude is calculated using

$$
\begin{aligned}
R_{k}= & \left\{\left(\sum_{n=1}^{N_{k}} R_{k, n} \cos \left(\phi_{k-1, n}+\varphi_{k, n}^{(I)}\right)\right)^{2}\right. \\
& \left.+\left(\sum_{n=1}^{N_{k}} R_{k, n} \sin \left(\phi_{k-1, n}+\varphi_{k, n}^{(I)}\right)\right)^{2}\right\}^{1 / 2}
\end{aligned}
$$

In the deterministic phase increment model, $M$ independent scatterers are uniformly distributed over the perimeter of each ellipse. For a given excess delay, phase of the first multipath component is generated according to $U[0,2 \pi)$ distribution. When the portable moves from point $k-1$ to point $k$ in space, the phase is updated using

$$
\phi_{k, n}=\phi_{k-1, n}+\varphi_{k, n}^{(I)}
$$

This process of phase updating in space continues until a path in the given excess delay (bin) ceases to exist. When a component at the same excess delay becomes present again a new set of randomly positioned scatterers are generated on the same ellipse using a uniform distribution. It should be emphasized that in this model the initial positions of scatterers on each ellipse are random, but updating of phase is deterministic; i.e., knowing $\theta_{0, n, k}, S_{n, m}$ and $\alpha_{k, n, m}$, the value of $\varphi_{k, n}^{(I)}$ in (12) is calculated deterministically. This is the reason for the notion "deterministic phase increment model." As a special limiting case the number of scatterers on each ellipse may be set at $M=1$. For this case (12) reduces to

$$
\begin{aligned}
\varphi_{k, n}^{(I)} & =\theta_{k, n, 1}-\theta_{k-1, n, 1} \\
& =\Delta \beta_{k, n, 1} \\
& =\frac{2 \pi}{\lambda}\left(\left|\overline{T S_{n, 1} P_{k}}\right|-\left|\overline{T S_{n, 1} P_{k-1}}\right|\right)
\end{aligned}
$$

and, subsequently, the relationship for updating phase (15) changes to

$$
\phi_{k, n}=\phi_{k-1, n}+\Delta \beta_{k, n, 1}
$$

which is quite clear from Fig. 1.

In the remainder of this section, the expression for parameters $p_{n}$ and $q_{n}$ of the $n$th ellipse (corresponding to the $n$th bin in a profile that has a path component) is provided (derivation appears in Appendix A)

$$
\begin{aligned}
& p_{n+1}=p_{n}+c \tau_{0} \\
& q_{n+1}=\sqrt{q_{n}^{2}+2 p_{n} c \tau_{0}+c^{2} \tau_{0}^{2}} .
\end{aligned}
$$

It should be emphasized that the model described in this section is a comprehensive multiple reflector phase model which does not have limitations of other simplified deterministic phase models.

\section{The Random Phase Increment Model}

In the random phase increment model (model II), the phase increment is a random variable, i.e., starting with a $U[0,2 \pi)$ initial phase, each subsequent phase value is obtained by adding a random phase increment to the previous phase value. Suppose $\phi_{k, n}$ and $\varphi_{k, n}^{(I I)}$ are the phase and the phase increment component, respectively, at the $n$th bin of the $k$ th profile. Path phase at the $n$th bin of the $(k+1)$ st profile is given by

$$
\phi_{k+1, n}=\phi_{k, n}+\varphi_{k, n}^{(I I)}
$$

where superscript (II) is used to indicate model II. Phase updating mechanism of (19) can also be written with reference to the initial path phase $\phi_{0, n}$; i.e.,

$$
\phi_{k+1, n}=\phi_{0, n}+\sum_{i=0}^{k} \varphi_{i, n}^{(I I)} .
$$

$$
\varphi_{k, n}^{(I)}=\tan ^{-1}\left(\frac{\sum_{m_{1}=1}^{M} \sum_{m_{2}=1}^{M} \alpha_{k, n, m_{1}} \alpha_{k, n, m_{2}} \sin \left(\theta_{k, n, m_{1}}-\theta_{k, n, m_{2}}\right)}{\sum_{m_{1}=1}^{M} \sum_{m_{2}=1}^{M} \alpha_{k, n, m_{1}} \alpha_{k, n, m_{2}} \cos \left(\theta_{k, n, m_{1}}-\theta_{k, n, m_{2}}\right)}\right)
$$


Using the following notation:

$$
\zeta_{k, n}=\sum_{i=0}^{k} \varphi_{i, n}^{(I I)}
$$

the phase updating relation (20) changes to

$$
\phi_{k+1, n}=\phi_{0, n}+\zeta_{k, n}
$$

The distribution of phase tends to Gaussian, following the central limit theorem. Generally the parameters of probability distribution of this increment are functions of $s_{k} / \lambda$. As an example, $\varphi_{k, n}^{(I I)}$ can be assumed to be a Gaussian random variable with zero mean and standard deviation $\sigma_{s / \lambda}$. By making $\sigma_{s / \lambda}$ an increasing function of $s / \lambda$ ( or $s$, for a fixed $\lambda$ ), the degree of correlation between $\phi_{k-1, n}$ and $\phi_{k, n}$ can be controlled. For $s=0$, $\sigma_{s / \sigma}=0$, and $\theta_{k-1}=\theta_{k}$ (assuming space-invariant channel). The correlation between $\phi_{k-1, n}$ and $\phi_{k, n}$ decreases as $\sigma_{s / \lambda}$ increases until they become uncorrelated. In the phase increment model described in this paper, $\varphi^{(I I)}\left(s_{k} / \lambda\right)$ is assumed to be a zero-mean Gaussian random variable with functional form of standard deviation given by

$$
\sigma_{s / \lambda}=\sigma_{\max }\left(1-e^{-b(s / \lambda)}\right)
$$

$\sigma_{\max }$ and $b$ are controlling parameters, the values of which determine degree of correlation on spatially adjacent phase components. The random phase increment model with the same functional form of phase increment [Gaussianly distributed increment $\left.\varphi\left(s_{k} / \lambda\right)\right]$ has been previously used in the simulation of the phase components of a wide-band mobile radio channel [23].

As in model I, in this model the phase updating process in space is interrupted when a path component ceases to exist at a given bin. A new $U[0,2 \pi)$ distributed phase is generated if a path with the same excess delay appears at a later profile, and random updating procedure starts again.

\section{Results OF THE MODELING}

In this section, performances of the two phase models described above are evaluated by means of extensive computer simulations. The results of simulations are compared with those of the empirical wide-band database described in Section II.

\section{A. General Comments}

In order to study the validity and suitability of each phase model, sequences of impulse response profiles were generated employing empirical path arrival times and path amplitudes, and simulated phase values. The simulated phases were obtained using each model under diversified sets of conditions. Cumulative probability distribution function (CDF) and other statistical properties of the empirical and simulated phase increments were obtained and compared. Furthermore, a CW envelope fading database was set up by vector addition of all multipath components of each impulse response profile (13). The number of empirical impulse response profiles measured at each location was
75 , with a sampling distance of $2 \mathrm{~cm}$. Each segment of CW envelope fading waveforms, therefore, spans a length of $150 \mathrm{~cm}$ in space. The CDF, level crossing rate (LCR), average duration of fades (ADF's), as well as Doppler spectra of simulated and measured $\mathrm{CW}$ envelope fading data were obtained and compared. In the following, the results for each model are reported separately, and then compared.

\section{B. Results for Model I}

As described in Section III, in the deterministic phase increment model, each multipath component results from reflection by $M$ independent uniformly distributed random scatterers positioned around a given ellipse. Meanwhile, reflection coefficients corresponding to scatterers were assumed to be independent random variables with a uniform $[0,1]$ distribution.

The simulations were performed using one, two, five, or ten scatterers on the perimeter of each ellipse. CW fading waveforms were generated, and their Doppler spectra and CDF's were obtained and compared with the corresponding empirical curves. Portion of the results are reproduced in Fig. 2. It should be noted that in applying the deterministic phase increment model if the number of scatterers on each ellipse $M$ is even, half of scatterers are distributed on the upper half ellipse and half on the lower half ellipse. If $M$ is odd, one-half ellipse will have one more scatterer than the other half ellipse. Results of extensive simulations show that $M=5$ (three scatterers on the upper half ellipse and two on the lower half ellipse) produce reasonably accurate phase values.

In order to analyze the model, for each location a number of intermediate impulse response profiles were generated in between each of 75 wide-band profiles. The number of generated profiles $L$ changes the sampling distance to $D / L$. Since empirical profiles were recorded at a sampling distance of $2 \mathrm{~cm}$, profiles generated in between empirical samples were assumed to have the arrival time and amplitude sequences of their preceding empirical profile; only the phase sequences were updated at each point using the phase models. Due to high correlation on amplitude and arrival times at a sampling distance of $2 \mathrm{~cm}$ (reported in [2]), this approach is justified. It should be mentioned that phase is critically sensitive to path length and therefore it should be updated more frequently. The number of profiles generated between empirical profiles was set at 1, 4, 5, and 20. The corresponding sampling distances are $1 \mathrm{~cm}, 5,4$, and $1 \mathrm{~mm}$, respectively. To save space only part of the results are reproduced in Fig. 3. Inspection of these figures indicates that a sampling distance of $2 \mathrm{~cm}$ is sufficient to record main features of the channel.

Each empirical data record (impulse responses collected in one location) is $1.5 \mathrm{~m}$ long. In two other simulation approaches, each such record was divided into three segments, each $50 \mathrm{~cm}$ long and five segments each $30 \mathrm{~cm}$ long. (Wavelength of transmission is $30 \mathrm{~cm}$.) $\mathrm{CW}$ fading characteristics were obtained for each new shorter data segments. To save space only part of the results are reproduced in this paper. Fig. 4 shows firstand second-order statistics of the empirical and simulated CW fading waveforms when all data for both buildings are combined. Inspection of plots depicted in Fig. 4 indicates that firstand second-order statistics of CW fading waveforms of model I 

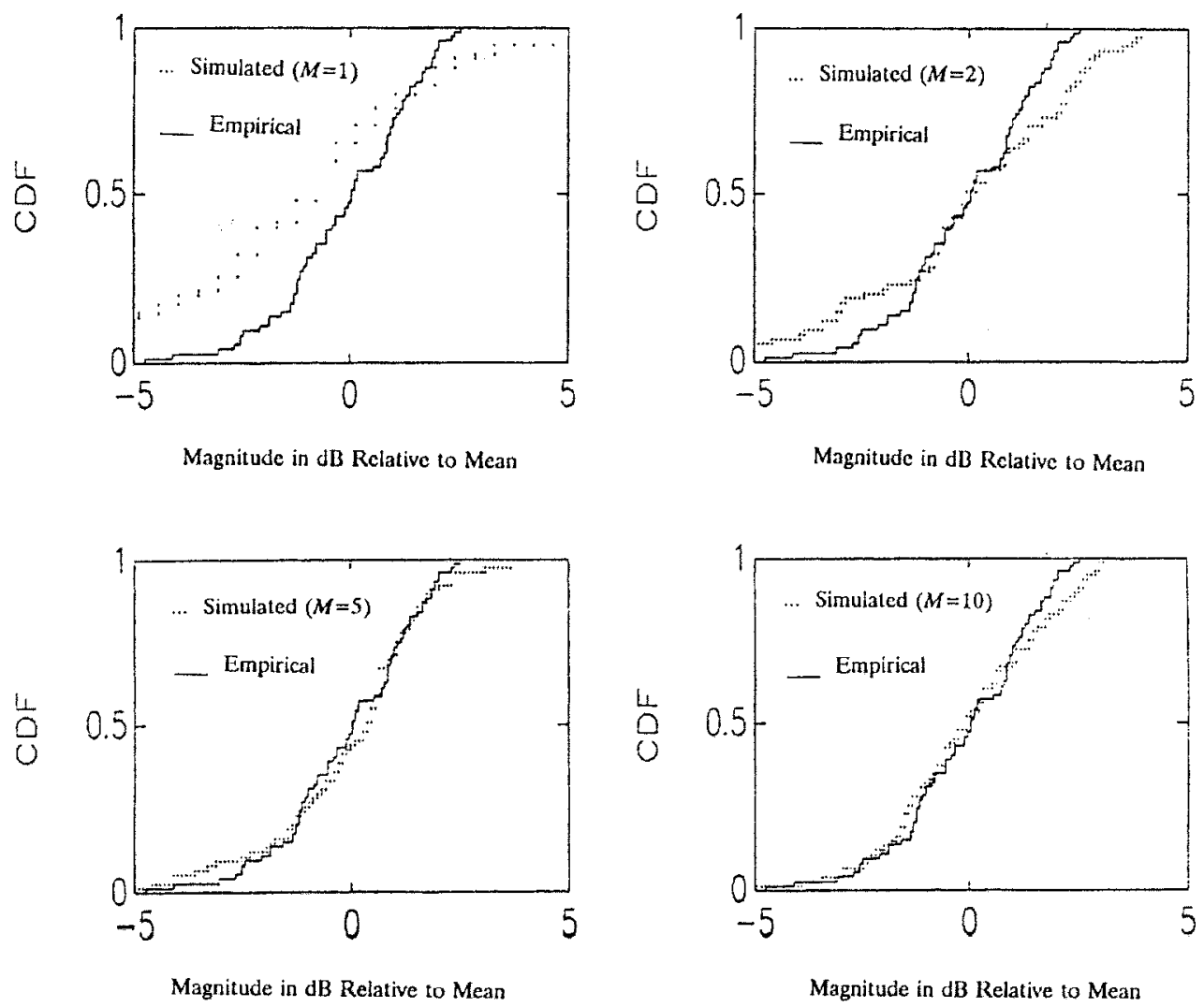

Fig. 2. CDF of CW waveforms of empirical data and model I for Bldg. B, antenna separation $10 \mathrm{~m}$, location 1, with one, two, five, and ten scatterers.
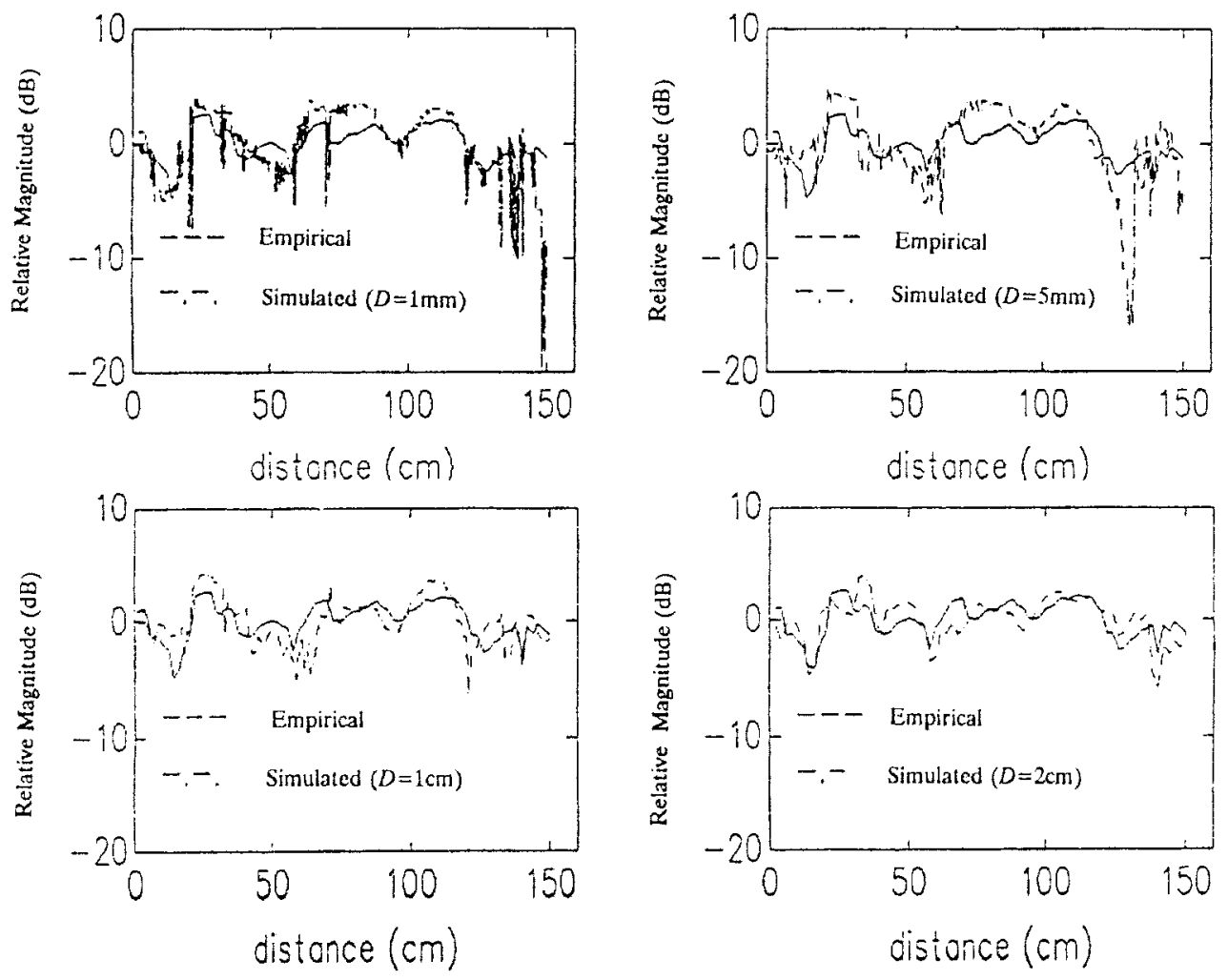

Fig. 3. CW fading waveforms of empirical data and model I. Bldg. B, antenna separation $10 \mathrm{~m}$, location 1, with sampling distance of 1 and $5 \mathrm{~mm}$ and 1 and $2 \mathrm{~cm}$.

show very good agreement with those obtained from measurements. Furthermore, comparison of the large number of simu- lated and empirical data records confirms applicability of the deterministic phase increment model. 

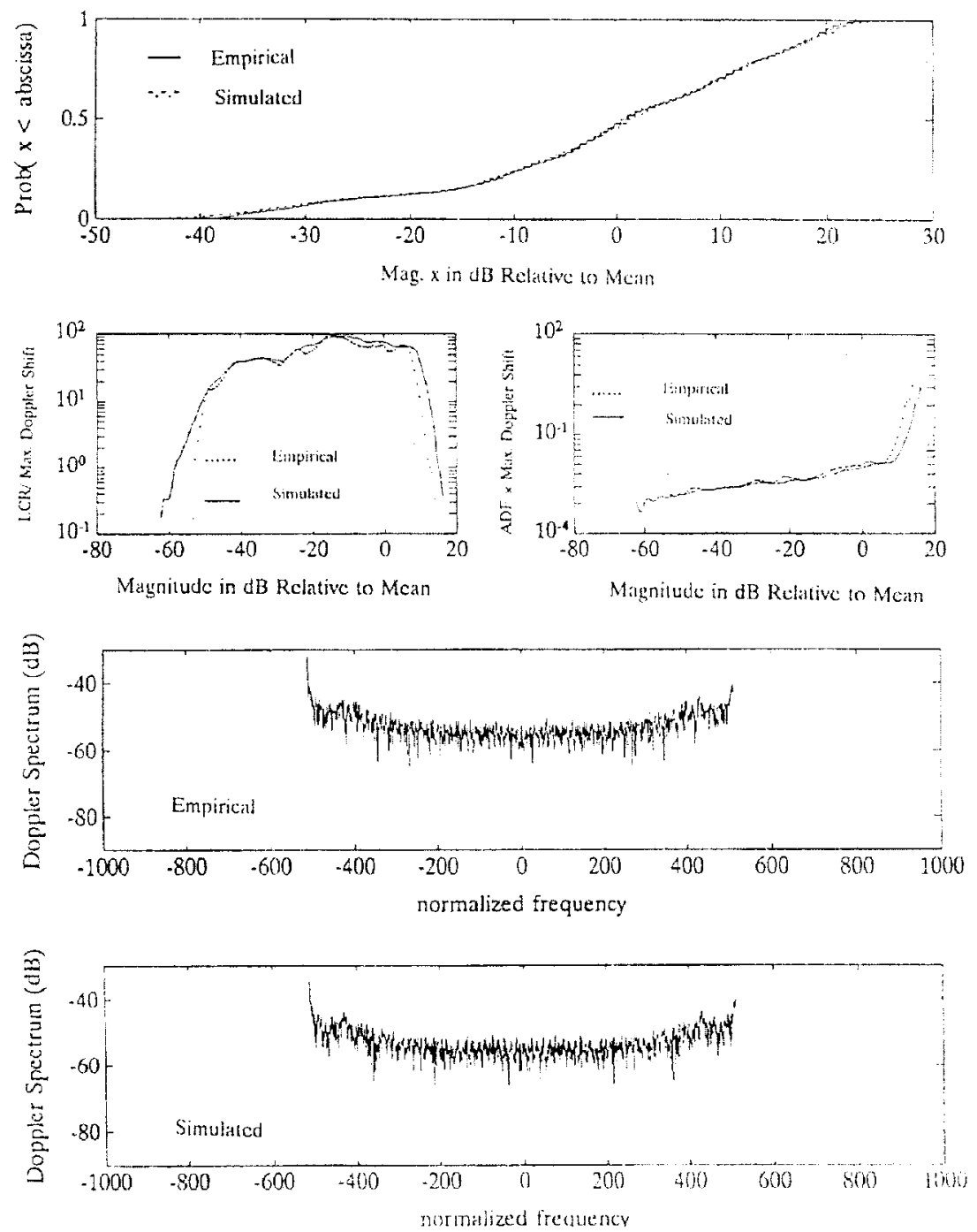

Fig. 4. CDF, LCR, ADF, and Doppler spectra of CW fading of empirical and model I simulated along a track of $150 \mathrm{~cm}$. Data for all antenna separations of both buildings are combined. Portable speed assumed to be $25 \mathrm{~cm} / \mathrm{s}$.

\section{Results for Model II}

In the random phase increment model, for each portable position in space a Gaussianly distributed phase increment was generated and used to update phase. The functional form of standard deviation of the phase increment is given in (23). As mentioned earlier $\sigma_{\max }$ and $b$ are controlling parameters. Their values determine degree of correlation on spatially adjacent phase components. Numerical values $\sigma_{\max }=10^{\circ}$ and $b=1$ obtained in similar modeling for the mobile channel [23] were used in this work. In [23], the values of parameters were obtained by computer simulation using the following approach: over local (small) areas the phase components should exhibit the right degree of spatial correlation, while over global (large) areas their distribution should approach that of a $U[0,2 \pi)$ random variable. It should he noted that wavelength of transmission in [23] is also around $30 \mathrm{~cm}$.

Again, for an exact analysis of this model the total area covered by the portable in each location is divided to three segments, each covering $50 \mathrm{~cm}$ as well as five segments each covering one wavelength $(30 \mathrm{~cm})$. The CDF, LCR, ADF, and Doppler spectrum of the empirical and simulated CW envelope fading data were obtained and compared. Firstand second-order statistics of $\mathrm{CW}$ fading data (empirical and simulated), when all data of two buildings are combined are shown in Fig. 5. Detailed study of fading statistics generated by this model provides excellent agreement with empirical data. Again, the large database used for performance evaluations confirms the validity and applicability of this model.

Meanwhile, extensive simulation of model I when the number of scatterers becomes large has also been carried out. Results show a good agreement between models I and II. For example simulation shows that the average standard deviation of phase increments of model I (with five scatterers) is $5.8^{\circ}$. In model II the standard deviation of phase increments is not constant and changes with the position of the portable. Averaging the standard deviation of phase increments of model II, i.e., (23), over the distance traveled by the portable, i.e., $5 \lambda=150 \mathrm{~cm}$, gives $6.32^{\circ}$ which is consistent with the value obtained from simulations of model I. 

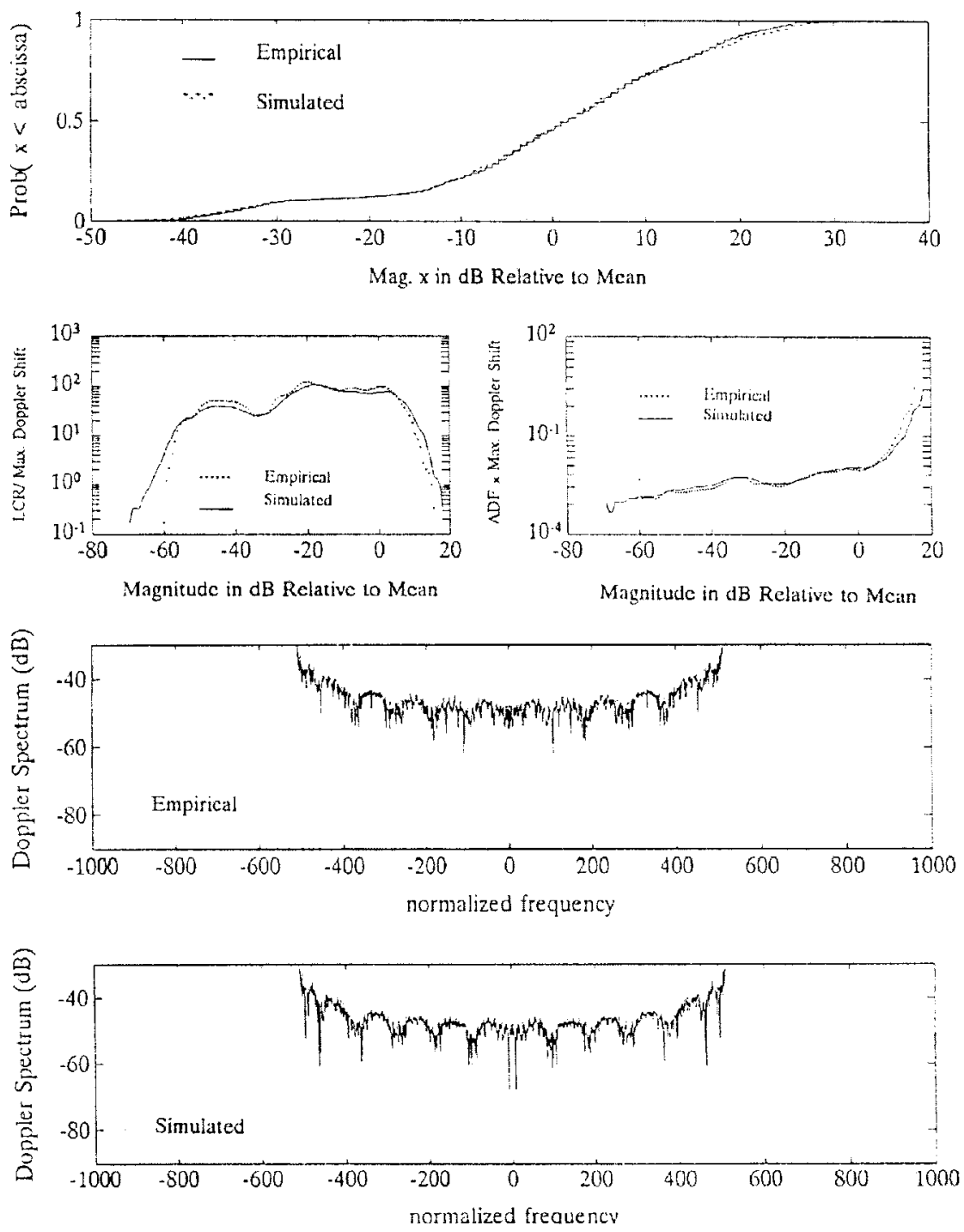

Fig. 5. CDF, LCR, ADF, and Doppler spectra of CW fading of empirical and model II simulated along a track of $150 \mathrm{~cm}$. Data for all antenna separations of both buildings are combined. Portable speed assumed to be $25 \mathrm{~cm} / \mathrm{s}$.

\section{Comparative Evaluations}

\section{A. Comparisons with Empirical Data}

A comparison was also made between path phase distribution of each model with those of the measured data. A single bin was considered and it was assumed that a path is present in that bin over all profiles. In model I, according to (12) and (15), $\phi_{0, n}$ is a $U[0,2 \pi)$ distributed random variable and $\varphi_{k, n}$ is a deterministic phase increment. The pdf of $\varphi_{k, n}$ is a Dirac delta function located at the calculated value of $\varphi_{k, n}$. Therefore, the resultant path phase has a pdf which is the convolution of a $U[0,2 \pi)$ density function with $\delta\left(\varphi_{k, n}\right)$. The resultant is a $U[0,2 \pi) \mathrm{pdf}$ shifted by the value of $\varphi_{k, n}$. Removing modulo $2 \pi$, the pdf of $\phi_{k, n}$ will be uniform.

Referring to (20)-(22), in model II initial phase $\phi_{0, n}$ has a $U[0,2 \pi)$ distribution; $\zeta_{k, n}$ is the summation of several independent zero-mean Gaussian increments with the functional form of standard deviation given in (23). According to (21) then, the mean value of $\zeta_{k, n}$ is zero and its variance is given by

$$
\sigma_{\zeta}^{2}=\sum_{i=0}^{k} \sigma_{\max }^{2}\left(1-e^{-b\left(s_{i} / \lambda\right)}\right)^{2}
$$

With the derivation given in Appendix B, (24) reduces to

$$
\begin{aligned}
\sigma_{\zeta}^{2}=\sigma_{\max }^{2} \cdot(k & +1+\frac{1-e^{-2 b D(k+1) / \lambda}}{1-e^{-2 b(D / \lambda)}} \\
& \left.-2 \frac{1-e^{-b D(k+1) / \lambda}}{1-e^{-b(D / \lambda)}}\right)
\end{aligned}
$$

Therefore, if $k$ is large one can assume $\zeta_{k, n}$ to be a zero-mean Gaussianly distributed random variable with the variance given in (25). Now considering (22), due to independence of $\phi_{0, n}$ and 


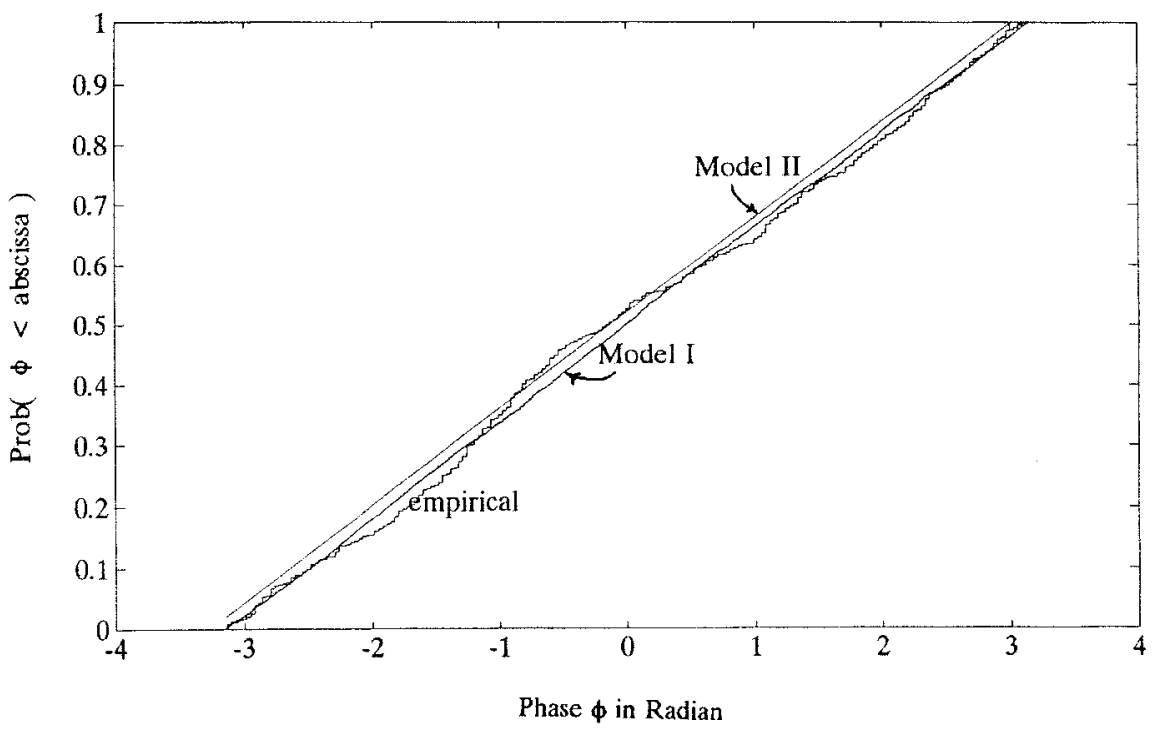

Fig. 6. CDF of path phases of empirical data and simulated models I and II. Bldg. A, antenna separation $30 \mathrm{~m}$, location 15.

$\zeta_{k, n}$, pdf of $\phi_{k, n}$ will be the convolution of pdf of $\phi_{0, n}$ [which is $U[0,2 \pi)]$ with the distribution of $\zeta_{k, n}$ (which is Gaussian). The final result is (derivation in Appendix C)

$$
\begin{aligned}
P\left[\phi_{k, n}<\xi\right]= & \frac{1}{2 \pi} \int_{-\pi}^{\xi}\left(Q\left(\frac{y-\pi}{\sigma}\right)-Q\left(\frac{y+\pi}{\sigma}\right)\right) d y \\
& \cdot[\bmod (2 \pi)], \quad-\pi \leq \xi<\pi
\end{aligned}
$$

where

$$
Q(y)=\frac{1}{\sqrt{2 \pi}} \int_{y}^{\infty} e^{-u^{2} / 2} d u .
$$

In Fig. 6, the cumulative distribution functions of path phases for both models and for empirical data are shown. This figure shows consistency and good fit between the distribution of path phases of both models with each other and with empirical data.

\section{B. Computation Efficiency of Each Model}

Before commenting on computation efficiency of the models, it should be emphasized again that in both models chains of spatially correlated phase values are interrupted when a path at a given excess delay (bin) ceases to exist. A new chain of phase values, with uniformly distributed first component starts if a path with the same excess delay appears at a later profile. It has been shown in [2] that amplitudes of multipath components become essentially uncorrelated at spatial separations greater than $10 \mathrm{~cm}$. Assuming the same degree of correlation on path arrival times, on the average, every $10 \mathrm{~cm}$ of space the sequence of correlated phases are interrupted. Assuming a sampling distance of $2 \mathrm{~cm}$, on the average after each $K_{1}=10 \mathrm{~cm} / 2 \mathrm{~cm}=5$ times of phase updating the sequence is interrupted and new phase components with new scatterers are generated. In the random phase increment model phase of the first component is selected according to $U[0,2 \pi)$ distribution and is updated for the next
$\left(K_{1}-1\right)$ components. In model II denoting the number of computations required to generate each uniformly distributed initial phase value by $G_{2}$ and the number of computations required to update each path phase value by $U_{2}$ the average number of computations for all excess delays and all profiles will be

$$
N C_{\text {model II }}=\left[G_{2}+\left(K_{1}-1\right) U_{2}\right] N_{a v} \frac{K}{K_{1}}
$$

where $N_{a v}$ is the average number of multipath components in each profile. In model I, phase of the first path is generated according to a $U[0,2 \pi)$ distribution and is updated based on (15). In this regard, the number of computations for generating $M$ scatterers on each ellipse and for generation of $M$ attenuation coefficients $\left(\alpha_{k, n, m}\right)$ should be added to the number of computations required to update $\left(K_{1}-1\right)$ path phases [based on (12)]. Denoting the number of computations needed to generate initial phases by $G_{1}$ and the number of computations required to update phase in model I with $U_{1}$, the average number of computations for all excess delays of all profiles in model I is

$$
N C_{\text {model I }}=\left[G_{1}+M G_{1}+M G_{1}+\left(K_{1}-1\right) U_{1}\right] N_{a v} \frac{K}{K_{1}} .
$$

The ratio of the number of computations (NC) of two models, i.e., $t=N C_{\text {model II }} / N C_{\text {model I }}$, is used as a criteria to compare efficiency. When the number of scatterers in the deterministic phase increment model increases, $t$ decreases implying that model II is better. The ratio $t=1$ is obtained at $M=2$. Therefore, with two scatterers the two models have similar efficiency. By considering only one scatterer on each confocal ellipse, the deterministic phase increment model is more efficient than the random phase increment model. If $M$ exceeds two, the random phase increment model is more efficient. In model I with five scatterers the exact value of $t^{-1}$ is 1.67 , implying that the random phase increment model is 1.67 times faster (more efficient) than the deterministic phase model. 
It has been shown in the previous section that for right choice of parameters both models I and II are capable of producing reliable estimates of signal phase. The above comparison, however, shows that for generating a large number of phase components typical in simulation applications, the random phase increment model is computation-wise more efficient and therefore more practical.

\section{Simulation Algorithms}

In the previous section, the two phase models were described in detail. The models were incorporated into a simulation tool that generates consistent sets of phase values for multipath components of consecutive impulse response profiles. Spatial correlation is imposed using models I or II. The user decides on the choice of the phase model. Simulation of the phase is performed according to the algorithms described in this section.

\section{A. Model I}

The simulation proceeds as follows: initial parameters of the model (antenna separation, sampling distance, number of scatterers $M$, wavelength of transmission $\lambda$, and binwidth $\tau_{0}$ ) are read into the program. Sequences of ones and zeros (path indicator sequence) for each profile are also read, where a one indicates presence of a multipath component in the given bin, and zero indicates absence of a path in the corresponding bin. For each one, in the first profile a $U[0,2 \pi)$ phase value is generated. For the $i$ th bin of the $m$ th profile $(m=2,3,4, \cdots)$, path phase is again generated according to $U[0,2 \pi)$ distribution if the $i$ th bin of the $(m-1)$ st profile does not contain a path. If a path appears in the $i$ th bin of the $(m-1)$ st profile, a corresponding ellipse is generated and $M$ scatterers are placed with a uniform distribution on its perimeter. Phase of the path at $i$ th bin of the $m$ th profile is then updated using (12) and (15). Since scatterers are made of different materials with different reflection properties, for each scatterer a $U[0,1)$ random attenuation coefficient is also generated. This updating process is repeated for the $i$ th bin of subsequent profiles until a path becomes absent in that bin. The process restarts with generation of new random independent scatterers on the same ellipse when a path component appears again at the $i$ th bin of a later profile. The phase of this initial component is again generated according to a $U[0,2 \pi)$ distribution.

It should be emphasized that a group of $M$ scatterers corresponding to the $i$ th bin of a profile is generated independently of the group of scatterers for the $j$ th bin. This is because path components at different excess delays are assumed to be reflected by independent set of scatterers.

\section{B. Model II}

Simulation algorithm for the random phase increment model is identical to the one proposed in [23]. After entering input parameters the simulation proceeds as follows. For every bin of the first profile which has a path, the phase is generated independently according to a $U[0,2 \pi)$ distribution. For a path in the $i$ th bin of the $m$ th profile (if such a path exists), where $m=2,3,4, \cdots$, the phase is generated according to a $U[0,2 \pi)$ distribution if there was no path in $i$ th bin of the $(m-1)$ st profile.

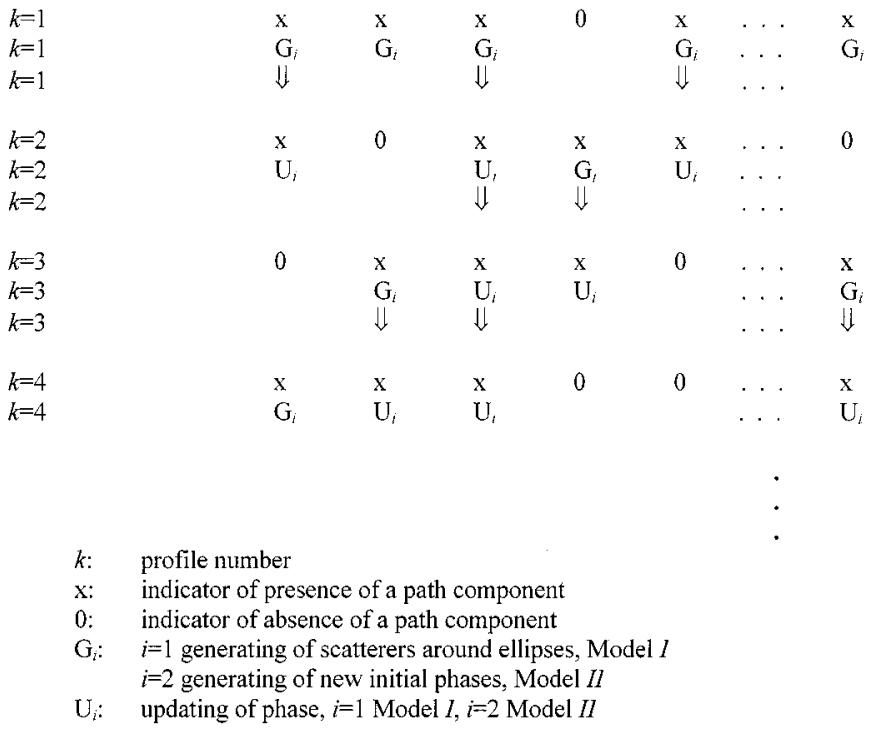

Fig. 7. Phase initiation and updating for both models.

If there was a path in the $i$ th bin of the $(m-1)$ st profile, however, a phase increment is generated according to a zero-mean Gaussian distribution with a standard deviation given in (23); then this normally distributed increment is added to the previous phase value to obtain current phase value [see (19)]. This process is repeated for all $N$ excess delays and all $K$ positions of portable. A graphical description of this algorithm is depicted in Fig. 7.

\section{CONCLUSION}

In this paper, two phase models for the wide-band indoor radio propagation channel were proposed and studied in great detail. In model I, phase of each path is updated deterministically using several random independently located scatterers for each multipath component. In model II, phase of each path is updated with random independent Gaussian increments whose standard deviations change with distance. Performance of these models were evaluated by means of extensive computer simulations and by utilization of a large database of 12000 impulse response profiles of the channel measured at two dissimilar office buildings. First- and second-order statistics of the narrow-band CW fading waveforms were obtained using simulated phases, and compared with those of empirical data. It was shown that model I with five independent scatterers for each path, and model II with appropriate choice of standard deviation of Gaussian increments both provide results consistent with empirical results. Multiple reflectors are considered explicitly in model I, while in model II it is implicitly included. Furthermore, the two phase models were compared with each other. Statistical properties of phase increments in model I were studied simulated and compared with increments of model II. It was shown that with five scatterers, phase increments of model I agree well with the normal distribution of phase increments of model II. Standard deviation of phase increments in model I shows good agreement with the standard deviation of phase increments of model II. Comparison of these models was also carried out from computational efficiency. It was shown that with five scatterers 
in model I, model II is 1.7 times faster (more efficient) than model I. Therefore, it is recommended to employ model II in the simulation of phases of the indoor radio propagation channel when large number of profiles are to be generated. Increasing the number of scatterers in model I makes this model more accurate, but less efficient and therefore less attractive for large simulation applications. The models and results reported in this paper can be used in the simulation and performance evaluation of indoor wireless communication systems.

\section{APPENDIX A}

As described in Section III-B, in the deterministic phase increment model the portable antenna is assumed to be initially located on the right focal point of confocal ellipses. A maximum number of $N$ groups of confocal ellipses are generated. Generation of ellipses depends on existence of a path component at the given excess delay. Referring to the equation of ellipses (9), with transmitter and receiver located at $\left(-\sqrt{ }\left(p_{n}^{2}-q_{n}^{2}\right), 0\right)$ and $\left(\sqrt{ }\left(p_{n}^{2}-q_{n}^{2}\right), 0\right)$, respectively, the distance between two consecutive ellipses [assuming path components exist in the $n$th and $(n+1)$ st bins $]$ is $c \tau_{0}\left(\tau_{0}\right.$ is binwidth and $c$ speed of light). Therefore

$$
p_{n+1}=p_{n}+c \tau_{0}
$$

due to confocality of ellipses we have

$$
q_{n+1}=\sqrt{q_{n}^{2}+2 p_{n} c \tau_{0}+c^{2} \tau_{0}^{2}} .
$$

It is assumed that with a maximum displacement of $K D$ the portable does not leave the first ellipse. Therefore, with the antenna separation $2 r$, parameters $p$ and $q$ of the first ellipse will be

$$
\begin{aligned}
p_{1} & =r+K D \\
q_{1} & =\sqrt{K D(K D+2 r)} .
\end{aligned}
$$

\section{APPENDIX B}

Derivation of (25).

Since the phase increments $\varphi_{k, n}^{(\mathrm{II})}$ are independent, variance of $\zeta_{k, n}$ is

$$
\sigma_{\zeta}^{2}=E\left[\zeta_{k, n}^{2}\right]=\sum_{i=0}^{k}\left[\sigma_{\varphi_{k, n}^{(\mathrm{II})}}\right]^{2}=\sum_{i=0}^{k} \sigma_{\max }^{2}\left(1-e^{-b s_{i} / \lambda}\right)^{2} .
$$

Substituting $i D$ for $s_{i}$ in (B.1) ( $D$ is sampling distance) results in

$$
\sigma_{\zeta}^{2}=\sum_{i=0}^{k} \sigma_{\max }^{2}\left(1-e^{-b D i / \lambda}\right)^{2} .
$$

Denoting $e^{-b D / \lambda}$ by $x$, (B.2) reduces to

$$
\sigma_{\zeta}^{2}=\sum_{i=0}^{k} \sigma_{\max }^{2}\left(1-x^{i}\right)^{2}=\sum_{i=0}^{k} \sigma_{\max }^{2}\left(1-2 x^{i}+x^{2 i}\right) .
$$

The second and third terms of (B.3) can be written as

$$
\begin{aligned}
\sum_{i=0}^{k} x^{i} & =\frac{1-x^{k+1}}{1-x} \\
\sum_{i=0}^{k} x^{2 i} & =\frac{1-x^{2(k+1)}}{1-x^{2}} .
\end{aligned}
$$

Substituting (B.4) in (B.3) gives (25).

\section{APPENDIX C}

Derivation of (26).

Since $\phi_{0, n}$ and $\zeta_{k, n}$ are independent, the pdf of the sum is the convolution of the pdf of each variable. Instead of convolving a $U[-\pi, \pi)$, with a zero-mean Gaussian pdf it is easier to convolve derivative of pdf $U[-\pi, \pi)$, which is two Dirac delta functions with weights $1 / 2 \pi$ and $-1 / 2 \pi$ located at $-\pi$ and $\pi$, respectively, with the Gaussian pdf and then integrate the result. Denoting the $U[-\pi, \pi)$ distribution of $\phi_{0, n}$ by $f_{1}(x)$, the zero-mean Gaussian distribution with variance $\sigma^{2}$, associated to $\zeta_{k, n}$ by $f_{2}(x)$ and the pdf of $\phi_{k, n}$ by $f(x)$, one obtains

$$
f(x)=\frac{1}{2 \pi} \int_{-\infty}^{x}\left(f_{2}(y+\pi)-f_{2}(y-\pi)\right) d y
$$

which reduces to

$$
f(x)=\frac{1}{2 \pi \sigma \sqrt{2 \pi}} \int_{-\infty}^{x}\left(e^{-(y+\pi)^{2} / 2 \sigma^{2}}-e^{-(y-\pi)^{2} / 2 \sigma^{2}}\right) d y .
$$

Denoting $(y+\pi) / \sigma$ by $u$ and $(y-\pi) / \sigma$ by $v($ C.2) reduces to

$$
\begin{aligned}
f(x)=\frac{1}{2 \pi}\left[\frac{1}{\sqrt{2 \pi}} \int_{-\infty}^{x+\pi / \sigma} e^{-u^{2} / 2} d u\right. \\
\left.-\frac{1}{\sqrt{2 \pi}} \int_{-\infty}^{x-\pi / \sigma} e^{-v^{2} / 2} d v\right]
\end{aligned}
$$

which is the same as

$$
\begin{aligned}
f(x) & =\frac{1}{2 \pi}\left[1-Q\left(\frac{x+\pi}{\sigma}\right)-1+Q\left(\frac{x-\pi}{\sigma}\right)\right] \\
& =\frac{1}{2 \pi}\left[Q\left(\frac{x-\pi}{\sigma}\right)-Q\left(\frac{x+\pi}{\sigma}\right)\right] .
\end{aligned}
$$

Removing modulo $2 \pi$, the CDF of $\phi_{k, n}$ reduces to (26).

\section{ACKNOWLEDGMENT}

The authors would like to thank the Electrical Engineering Department at the Sharif University of Technology and Telecommunications and the Traffic Control Systems Group at Delft University of Technology for the use of their facilities. 


\section{REFERENCES}

[1] H. Hashemi, "The indoor radio propagation channel," Proc. IEEE, vol. 81, pp. 943-968, July 1993.

[2] -, "Impulse response modeling of indoor radio propagation channels," IEEE J. Select. Areas Commun., vol. 11, pp. 967-978, Sept. 1993.

[3] H. Hashemi and D. Tholl, "Statistica1 modeling and simulation of the RMS delay spread of indoor radio propagation channels," IEEE Trans. Veh. Technol., vol. 43, pp. 110-120, Feb. 1994

[4] H. Nikookar and H. Hashemi, "Statistical modeling of signal amplitude fading of indoor radio propagation channels," in Proc. 2nd Int. Conf. Universal Personal Commun., Ottawa, Canada, Oct. 1993, pp. 84-88.

[5] T. S. Rappaport, "Characterization of UHF multipath radio channels in factory buildings," IEEE Trans. Antennas Propagat., vol. 37, pp. 1058-1069, Aug. 1989.

[6] T. S. Rappaport, S. Y. Seidel, and K. Takamizawa, "Statistical channel impulse response models for factory and open plan building radio communication system design," IEEE Trans. Commun., vol. 39, pp. 794-807, May 1991.

[7] S. Y. Seidel and T. S. Rappaport, "Simulation of UHF indoor radio channels for open plan building environments," in IEEE Proc. Veh. Technol. Conf.. Miami, FL, May 1990, pp. 597-602.

[8] D. M. J. Devarsivatham, "Time delay spread and signal level measurements of $850 \mathrm{MHz}$ radio waves in building environments," IEEE Trans. Antennas Propagat., vol. AP-34, pp. 1300-1305, Nov. 1986.

[9] —_ "A comparison of time delay spread and signal level measurements within two dissimilar office buildings," IEEE Trans. Antennas Propagat., vol. AP-35, pp. 319-324, Mar. 1987.

[10] - "Multiple time delay spread in the digital portable radio environment," IEEE Commun. Mag., vol. 25, pp. 13-21, June 1987.

[11] K. Pahlavan, R. Ganesh, and T. Hotaling, "Multipath propagation measurements on manufacturing floors at $910 \mathrm{MHz}$," Electron. Lett., vol. 25, pp. 225-227, Feb. 1989.

[12] K. Ganesh and K. Pahlavan, "Statistical modeling and computer simulation of indoor radio channel," Proc. Inst. Elec. Eng., vol. 138, pp. 153-161, June 1991.

[13] S. Howard and K. Pahlavan, "Measurement and analysis of the indoor radio channel in frequency domain," IEEE Trans. Instrum. Meas., vol. 39, pp. 751-755, Oct. 1990.

[14] R. J. C. Bultitude, "Measurement characterization and modeling of indoor $800 / 900 \mathrm{MHz}$ radio channels for digital communications," IEEE Commun. Mag., vol. 25, pp. 5-12, June 1987.

[15] R. J. C. Bultitude, S. A. Mahmoud, and W. A. Sullivan, "A comparison of indoor radio propagation characteristics at $910 \mathrm{MHz}$ and $1.75 \mathrm{GHz}$," IEEE J. Select. Areas Commun., vol. 7, pp. 20-30, Jan. 1989.

[16] A. A. M. Saleh and R. A. Valenzuela, "A statistical model for indoor radio multipath propagation," IEEE J. Select. Areas Commun., vol. 5, pp. 128-137, Feb. 1987.

[17] C. Loo, "Measurements and models of a land mobile satellite channel and their applications to MSK signals," IEEE Trans. Veh. Technol., vol. 35, pp. 114-121, Aug. 1987.

[18] M. Fattouche, L. Petherick, and A. Fapojuwo, "Diversity for mobile radio communications," in Proc. 15 Biennial Symp. Commun., Kingston, Canada, June 1990, pp. 196-199.

[19] M. Fattouche, G. Morrison, H Zaghloul, and L. Petherick, "Diversity for indoor radio communications," in Proc. 33rd Midwest Symp. Circuits Syst., Calgary, Canada, Aug. 12-14, 1990.

[20] G. L. Turin, "Communication through noisy random-multipath channels," in 1956 IRE Conven. Rec., pp. 154-166.

[21] G. L. Turin et al., "A statistical model of urban multipath propagation," IEEE Trans. Veh. Technol., vol. VT-21, pp. 1-9, Feb. 1972.

[22] H. Suzuki, "A statistical model for urban radio propagation," IEEE Trans. Commun., vol. COM-25, pp. 673-680, July 1977.

[23] H. Hashemi, "Simulation of urban radio propagation Channel," IEEE Trans. Veh. Technol., vol. VT-28, pp. 213-224, Aug. 1979.

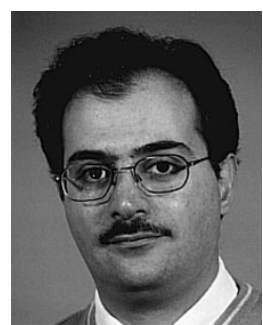

Homayoun Nikookar (M'92) received the B.S. and M.S. degrees (with distinction) in electrical engineering from Sharif University of Technology, Tehran, Iran, in 1986 and 1987, respectively, and the $\mathrm{Ph} . \mathrm{D}$. degree in electrical engineering from Delft University of Technology, Delft, The Netherlands, in 1995.

From 1988 to 1992, he served as a Lecturer in the Department of Electrical Engineering, AmirKabir University of Technology (Tehran Polytechnic Institute), Iran. From 1995 to 1998 , he was a Postdoctoral Researcher at the International Research Center for Telecommunications-Transmission and Radar, Delft University of Technology, where he is currently an Assistant Professor. His areas of interest include wireless channel modeling, multicarrier transmission, and CDMA.

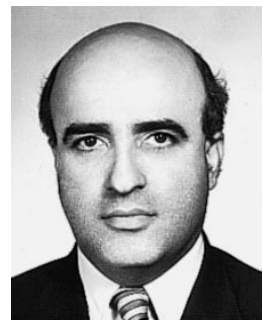

Homayoun Hashemi (M'89-SM'93) was born in Tehran, Iran. He received the B.S.E.E. degree from the University of Texas at Austin in 1972 and the M.S. and Ph.D. degrees in electrical engineering and computer science from the University of California at Berkeley, in 1974 and 1977, respectively. He also received the Master's degree in statistics from the University of California at Berkeley.

In the Summer of 1977, he served as a Consulting Engineer at Stanford Research Institute, Menlo Park, CA. From 1977 to 1979 , he was a Member of the Technical Staff at Bell Telephone Laboratories, Holmdel, NJ, where he was involved in system design for high-capacity mobile telephone systems. Since 1979, he has been a Faculty Member at Sharif University of Technology, Tehran, where he is currently a Full Professor of Electrical Engineering. He was on sabbatical leave at NovAtel Communications Ltd., Calgary, Alberta, Canada, during the 1990-1991 academic year, where he worked on various projects related to mobile and personal communications. The indoor radio propagation impulse response data collected under his supervision at NovAtel is still the largest of its kind in the world. He spent the Summers of 1992 and 1994 at the Electrical Engineering Department, University of Ottawa, Canada, where he was involved in propagation modeling at infrared frequencies for indoor wireless communications. In 1994, at the University of Ottawa he defined and supervised a project in which the largest database in the world on frequency responses of the indoor infrared channel was established and analyzed. In the Summer of 1993, he was a Visiting Researcher at TRLabs, Calgary, Canada, where he worked on wireless indoor radio communications. At TRLabs, he defined and supervised a project on temporal variations of the indoor radio propagation channel, in which again, the largest database of its kind in the world was set up and analyzed. He has done research on different aspects of wireless communications, including general system architecture, channel assignment, propagation modeling, performance analysis, and capacity evaluations for digital cellular, microcellular, and indoor wireless communication systems. His channel simulator package, SURP, developed in the 1970's has been used internationally in the design of digital cellular radio communication systems in the 1980's and 1990's. He also served as a Consultant to the Telecommunication Company of Iran and the Iranian Telecommunication Research Center and has contributed to different telecommunication expansion projects in Iran.

Dr. Hashemi is listed in Marquis Who's Who in the World and is one of four scientists in his field who has been awarded membership in the Iranian Academy of Science. 